\title{
BM-INDEPENDENCE AND CENTRAL LIMIT THEOREMS ASSOCIATED WITH SYMMETRIC CONES
}

\author{
JANUSZ WYSOCZAŃSKI \\ Institute of Mathematics, Wrockaw University \\ Pl. Grunwaldzki 2/4, 50-384 Wroctaw, Poland \\ E-mail: jwys@math.uni.wroc.pl
}

\begin{abstract}
We present a generalization of the classical central limit theorem to the case of noncommuting random variables which are bm-independent and indexed by a partially ordered set. As the set of indices I we consider discrete lattices in symmetric positive cones, with the order given by the cones. We show that the limit measures have moments which satisfy recurrences generalizing the recurrence for the Catalan numbers.
\end{abstract}

1. Introduction. In the classical Central Limit Theorem (CLT) one considers convergence of the normalized sums

$$
S_{n}:=\frac{1}{\sqrt{N}} \sum_{i=1}^{N} X_{i}
$$

of independent identically distributed random variables $X_{i}$, which are centered by the expectation $E\left(X_{i}\right)=0$ and with the variance $E\left(X_{i}^{2}\right)=1$. In this paper we consider a generalization of this to a non-commutative setting. Instead of the classical random variables, which are just functions on a probability space, we shall consider operators on some Hilbert space, and the classical independence will be replaced by the notion of bm-independence, defined in the next section. This notion is associated with partially ordered sets of indexes $\mathbf{I}$, instead of the totally ordered set $\mathbb{N}$ of positive integers. Hence we will have to replace the above summation over positive integers in the interval $[1, N]$ by a summation over some ascending family of finite subsets $\mathbf{J}_{N} \subset \mathbf{I}$. These sets have

2000 Mathematics Subject Classification: Primary 46L54; Secondary 81R50.

Key words and phrases: bm-independence, non-commutative central limit theorem, symmetric cones.

Research supported by KBN grant 1P03A01330, European Commission Marie Curie Host Fellowship for the Transfer of Knowledge "Harmonic Analysis, Nonlinear Analysis and Probability" MTKD-CT-2004-013389 and 7010 POLONIUM project "Non-Commutative Harmonic Analysis With Applications To Operator Spaces, Operator Algebras And Quantum Probability".

The paper is in final form and no version of it will be published elsewhere. 
to enjoy some geometrical properties of the ordinary intervals, so the natural candidates would be intervals in the partially ordered set I. However, our method of proof of the bm-Central Limit Theorem works better for some more particular choice of these sets. In addition, the classical expectation $E$ has to be replaced by a state $\varphi$, defined for our non-commutative random variables, and the convergence is considered as convergence of moments with respect to this state.

The notion of bm-independence is a generalization of the monotonic independence invented and studied initially by Muraki [5], and also of the boolean independence, which appeared first in the work by Bożejko [1] under the name generalized free product of states. The main generalization is by replacing the totally ordered set of indexes with partially ordered set indexing the non-commutative random variables. The basic example of the bm-independent family of (non-commutative) random variables is provided by the bm-extension construction [7]. This will be described also in section 3 . The construction is based on the ideas of [6], where a sequence of monotonically independent operators was constructed, providing example related to the work by Muraki. Another related construction was given by Lenczewski and Sałapata in [4].

2. bm-independence. Let $\mathbf{I}$ be a set partially ordered by a relation $\preceq$. For $\xi, \eta \in \mathbf{I}$ we shall write $\xi \prec \eta$ if and only if $\xi \preceq \eta$ and $\xi \neq \eta$. In a partially ordered set some elements $\xi, \eta \in \mathbf{I}$ may be non-comparable; in such case we shall write $\xi \nsim \eta$.

Let $\left\{\mathcal{B}_{\xi}: \xi \in \mathbf{I}\right\}$ be a family of subalgebras of a given algebra $\mathcal{B}$ and let $\varphi$ be a functional on $\mathcal{B}$.

Definition 1. We say that the algebras $\left\{\mathcal{B}_{\xi}: \xi \in \mathbf{I}\right\}$ are bm-independent in $\mathcal{B}$, with respect to the given functional $\varphi$ on $\mathcal{B}$, if the following two conditions hold:

BM1. If $\xi, \rho, \eta \in \mathbf{I}$ satisfy: $\xi \prec \rho \succ \eta$ or $\xi \prec \rho \nsim \eta$ or $\xi \nsim \rho \succ \eta$, then for any $b_{\xi} \in \mathcal{B}_{\xi}$, $b_{\rho} \in \mathcal{B}_{\rho}$ and $b_{\eta} \in \mathcal{B}_{\eta}$

$$
b_{\xi} b_{\rho} b_{\eta}=\varphi\left(b_{\rho}\right) b_{\xi} b_{\eta}
$$

BM2. If $\xi_{1}, \ldots, \xi_{n} \in \mathbf{I}$ satisfy $\xi_{1} \succ \cdots \succ \xi_{m} \nsim \cdots \nsim \xi_{k} \prec \cdots \prec \xi_{n}$, for some $1 \leq m \leq$ $k \leq n$, then for any $b_{\xi_{i}} \in \mathcal{B}_{\xi_{i}}$, with $1 \leq i \leq n$

$$
\varphi\left(b_{\xi_{1}} \ldots b_{\xi_{n}}\right)=\prod_{i=1}^{n} \varphi\left(b_{\xi_{i}}\right)
$$

If the set $\mathbf{I}$ is totally ordered, then these two conditions are just Muraki's conditions for monotonic independence. On the other hand, if the set $\mathbf{I}$ is totally disordered (no two elements are comparable) then the first condition is void, and the second one is the condition for boolean independence. The two conditions above are sufficient for the computation of the mixed moments, the expressions of the form $\varphi\left(b_{\xi_{1}} \ldots b_{\xi_{n}}\right)$, by means of the restrictions of the functional $\varphi$ to each subalgebra $\mathcal{B}_{\xi}$. In the next section we shall describe the construction of bm-independent subalgebras.

3. bm-product of Hilbert spaces and bm-extensions of operators. Let us consider a family $\left\{\mathbf{H}_{\xi}: \xi \in \mathbf{I}\right\}$ of Hilbert spaces, indexed by a partially ordered countable set $\mathbf{I}$, which have a common unit vector $\Omega$. One may think of a countable number of copies 
of $l^{2}$, with the natural orthonormal basis $\left\{\delta_{n}: n \geq 0\right\}$ and with $\Omega=\delta_{0}$; the orthonormal basis in $\mathbf{H}_{\xi}$ would then be $\left\{e_{\xi_{n}}=\delta_{n}: n \geq 0\right\}$ and $e_{\xi_{0}}=\Omega$. Let $\mathbf{H}_{\xi}^{0}$ be the orthogonal complement of $\Omega$ in $\mathbf{H}_{\xi}$.

Definition 2. The bm-product of Hilbert spaces $\left\{\mathbf{H}_{\xi}: \xi \in \mathbf{I}\right\}$ is the Hilbert subspace $\mathbf{H}$ of the free Fock space $\mathcal{F}_{\mathbf{I}}:=\mathcal{F}\left(\left\{\mathbf{H}_{\xi}: \xi \in \mathbf{I}\right\}\right)$ generated by these spaces, which is spanned by the vacuum vector $\Omega$ and the simple tensors of the form $h_{\rho_{j}} \otimes \cdots \otimes h_{\rho_{1}}$ with $\rho_{1} \prec \cdots \prec \rho_{j}$ and $h_{\rho_{i}} \in \mathbf{H}_{\rho_{i}}^{0}(1 \leq i \leq j)$. The orthogonal projection $\mathcal{F}_{\mathbf{I}} \mapsto \mathbf{H}$ will be denoted by $\mathbf{P}_{\mathbf{H}}$.

Let us assume that for each $\xi \in \mathbf{I}$ we are given an algebra $\mathcal{B}_{\xi}$ of operators bounded on $\mathbf{H}_{\xi}$.

Definition 3. The bm-extension of an operator $b_{\xi} \in \mathcal{B}_{\xi}$ onto $\mathbf{H}$ is defined as

$$
a_{\xi}:=\mathbf{P}_{\mathbf{H}} b_{\xi} \mathbf{P}_{\mathbf{H}}
$$

More explicitly, for $\rho_{1} \prec \cdots \prec \rho_{j} \in \mathbf{I}$

$$
a_{\xi}\left(h_{\rho_{j}} \otimes \cdots \otimes h_{\rho_{1}}\right)=0
$$

if $\xi \prec \rho_{j}$ or $\xi \nsim \rho_{j}$;

$$
a_{\xi}\left(h_{\rho_{j}} \otimes \cdots \otimes h_{\rho_{1}}\right)=\beta h_{\rho_{j-1}} \otimes \cdots \otimes h_{\rho_{1}}+f_{\xi} \otimes h_{\rho_{j-1}} \otimes \cdots \otimes h_{\rho_{1}}
$$

if $\rho_{j}=\xi$ and $b_{\xi} h_{\xi}=\beta \Omega+f_{\xi}$ with $f_{\xi} \perp \Omega$;

$$
a_{\xi}\left(h_{\rho_{j}} \otimes \cdots \otimes h_{\rho_{1}}\right)=\alpha h_{\rho_{j}} \otimes \cdots \otimes h_{\rho_{1}}+g_{\xi} \otimes h_{\rho_{j}} \otimes \cdots \otimes h_{\rho_{1}}
$$

if $\xi \succ \rho_{j}$ and $b_{\xi} \Omega:=\alpha \Omega+g_{\xi}$, with $g_{\xi} \perp \Omega$.

The bm-extension operators are bm-independent with respect to the vacuum state $\varphi(a):=\langle a \Omega \mid \Omega\rangle([7])$.

THEOREM 4. If $\mathcal{A}_{\xi}$ is the algebra of the bm-extension operators of the given algebra $\mathcal{B}_{\xi}$, then the algebras $\left\{\mathcal{A}_{\xi}: \xi \in \mathbf{I}\right\}$ are bm-independent with respect to the functional $\varphi$.

4. Positive symmetric cones and examples of discrete sublattices. Partial orders are related to positive cones in euclidian spaces in a natural way. If $V$ is a euclidian space and $\Pi \subset V$ is a positive cone, i.e. it is closed under addition of vectors and under multiplication by positive scalars, then it defines a partial order $\preceq$ on $V$ :

Definition 5. If $u, v \in V$ then $u \preceq v$ if $v-u \in \Pi$.

Since we seek the replacement of the set $\mathbb{N}$ of positive integers, which itself is a discrete lattice in the positive cone $\Pi=[0,+\infty)$ of $V=\mathbb{R}$, we shall consider analogous discrete lattices $\mathbf{I}$ in more general situations. In particular, we shall show in each of our Examples what are the replacements $J_{N}$ of the intervals $[1, N] \subset \mathbb{N} \subset[0,+\infty)$. Of course in a partially ordered set we can always consider intervals, which are defined as follows: if $\xi \prec \eta \in \mathbf{I}$ then $[\xi, \eta]:=\{\rho \in \mathbf{I}: \xi \preceq \rho \preceq \eta\}$. However, our methods of proof of the bm-Central Limit Theorems require a little more sophisticated definitions. In particular, we shall exhibit also some subsets $I_{\mathbf{k}} \subset J_{\mathbf{N}}$, which play a combinatorial and geometrical role in computing the limit recurrences (with the exception of Example 1).

The main examples we shall consider will be the following ( $d$ is a positive integer). 
ExAmple 1. Let $V:=\mathbb{R}^{d}$ and $\Pi:=\left(\mathbb{R}_{+} \cup 0\right)^{d}$, then $\mathbf{I}=\mathbf{I}_{d}:=\mathbb{N}^{d}$ and for $\mathbf{N}:=$ $\left(N_{1}, \ldots, N_{d}\right) \in \mathbf{I}_{d}$ we define the partial order $\preceq$ as

$$
\left(k_{1}, \ldots, k_{d}\right) \preceq\left(m_{1}, \ldots, m_{d}\right) \quad \text { if } \quad k_{1} \leq m_{1}, \ldots, k_{d} \leq m_{d}
$$

and the analogues of the intervals as

$$
J_{\mathbf{N}}:=\left\{\left(n_{1}, \ldots, n_{d}\right) \in \mathbf{I}_{d}: n_{1} \leq N_{1}, \ldots, n_{d} \leq N_{d}\right\}
$$

These sets are in fact lattice intervals in the partial order.

ExAmple 2. Let $V$ be the Minkowski spacetime and let $\Pi$ be the Lorentz light cone defined as

$$
\Pi:=\left\{\left(\mathbf{x} ; y_{1}, \ldots, y_{d}\right) \in \mathbb{R}_{+} \times \mathbb{R}^{d}: \mathbf{x}^{2} \geq y_{1}^{2}+\cdots+y_{d}^{2}\right\}
$$

and the partial order $\preceq$ is given by

$$
\left(\mathbf{x} ; y_{1}, \ldots, y_{d}\right) \preceq\left(\mathbf{z} ; w_{1}, \ldots, w_{d}\right) \quad \text { if } \quad \mathbf{z}-\mathbf{x} \geq\left(\sum_{i=1}^{d}\left(w_{i}-y_{i}\right)^{2}\right)^{\frac{1}{2}} .
$$

Then we define

$$
\mathbf{I}_{d}:=\left\{\left(\mathbf{k} ; m_{1}, \ldots, m_{d}\right) \in \mathbb{N} \times \mathbb{Z}^{d}: \mathbf{k}^{2} \geq m_{1}^{2}+\cdots+m_{d}^{2}\right\} \subset \Pi
$$

and for positive integers $\mathbf{N} \in \mathbb{N}$ we consider

$$
J_{\mathbf{N}}:=\left\{\left(\mathbf{k} ; m_{1}, \ldots, m_{d}\right) \in \mathbf{I}_{d}: \mathbf{k} \leq \mathbf{N}\right\} .
$$

In particular, the elements with the same time-coordinate $k$ are non-comparable. In this example

$$
I_{\mathbf{k}}:=\left\{\left(\mathbf{k} ; m_{1}, \ldots, m_{d}\right) \in \mathbf{I}_{d}\right\} .
$$

EXAMPLE 3. Let $V=\operatorname{Herm}_{d \times d}(\mathbb{R})$ be the vector space of $d \times d$ symmetric matrices with real entries, and let $\Pi \subset V$ be the positive cone of positive definite real symmetric matrices. Then we define

$$
\mathbf{I}_{d}:=\left\{\left(a_{i j}\right)_{i, j=1}^{d} \in \Pi: a_{i j} \in \mathbb{Z}\right\}
$$

the lattice of real symmetric positive definite matrices with integral entries, and for $\mathbf{N}=\left(N_{1}, \ldots, N_{d}\right)$ we set

$$
J_{\mathbf{N}}:=\left\{\left(a_{i j}\right)_{i, j=1}^{d} \in \mathbf{I}_{d}: a_{i i} \leq N_{i} \quad \forall 1 \leq i \leq d\right\}
$$

and

$$
I_{\mathbf{k}}:=\left\{\left(a_{i j}\right)_{i, j=1}^{d} \in \mathbf{I}_{d}: a_{i i}=k_{i} \quad \forall 1 \leq i \leq d\right\}
$$

if $\mathbf{k}=\left(k_{1}, \ldots, k_{d}\right)$.

EXAMPLE 4 . Let $\mathbf{I}_{d}$ be the set of vertices of a homogeneous rooted tree of degree $d \geq 2$. If $\xi_{0}$ is the root, and if $\operatorname{dist}(\rho, \eta)$ is the distance of $\rho$ and $\eta$, then for $\mathbf{N} \in \mathbb{N}$ we define

$$
\begin{gathered}
J_{\mathbf{N}}:=\left\{\xi \in \mathbf{I}_{d}: \operatorname{dist}\left(\xi_{0}, \xi\right) \leq N\right\}, \\
I_{\mathbf{k}}:=\left\{\xi \in \mathbf{I}_{d}: \operatorname{dist}\left(\xi_{0}, \xi\right)=\mathbf{k}\right\} .
\end{gathered}
$$

The partial order $\preceq$ is defined by "being on the geodesic closer to the root":

$$
\rho \preceq \eta \quad \text { if } \operatorname{dist}\left(\xi_{0}, \eta\right)=\operatorname{dist}\left(\xi_{0}, \rho\right)+\operatorname{dist}(\rho, \eta) .
$$


In particular, different elements which are at the same distance from the root are noncomparable.

REMARK 6 . The sets $J_{\mathbf{N}}$ are chosen so that they satisfy the following geometric property: if $\xi \in J_{\mathbf{N}}$ then the set $\left\{\eta \in J_{\mathbf{N}}: \xi \preceq \eta\right\}$ is a translation by $\xi$ of some other set $J_{\mathbf{K}}$, for properly chosen $\mathbf{K}$.

5. bm-Central Limit Theorems. Our bm-Central Limit Theorems have the following formulation (cf. [7] and [8]). Let $\left\{a_{\xi}: \xi \in \mathbf{I}_{d}\right\}$ be a family of bm-extension self-adjoint operators on $\mathbf{H}$ and let $\mathbf{I}_{d}, J_{\mathbf{N}}$ be defined as in section 4 . Let $\varphi$ be the vacuum state and let us assume that $\varphi\left(a_{\xi}\right)=0$ and $\varphi\left(a_{\xi}^{2}\right)=1$ for each $\xi \in \mathbf{I}_{d}$. For each index $\mathbf{N}$ as above let us define the normalized partial sums as follows:

$$
S_{\mathbf{N}}:=\frac{1}{\sqrt{\left|J_{\mathbf{N}}\right|}} \sum_{\xi \in J_{\mathbf{N}}} a_{\xi}
$$

These operators are self-adjoint, and since $\varphi$ is a vacuum state and $\varphi\left(\left(S_{\mathbf{N}}\right)^{0}\right)=1$, the moment sequence $\varphi\left(\left(S_{\mathbf{N}}\right)^{n}\right)$ is a positive definite sequence of real numbers, so if it converges to some $\alpha_{n}$ when $\mathbf{N} \rightarrow \infty$, then the limit sequence $\alpha_{n}$ is also positive definite.

Using the combinatorial reduction, as in [7] and [8], one can show that the odd moments of the $S_{\mathbf{N}}$ tend to zero, as $\mathbf{N} \rightarrow \infty$ :

$$
0=\lim _{\mathbf{N} \rightarrow \infty} \varphi\left(\left(S_{\mathbf{N}}\right)^{2 n+1}\right) .
$$

With the same method of combinatorial reduction, and using geometrical properties of the sets $\mathbf{J}_{\mathbf{N}}$, one can show the following for the even moments.

THEOREM 7. For each non-negative integer $n$ the limit

$$
g_{n}:=\lim _{\mathbf{N} \rightarrow \infty} \varphi\left(\left(S_{\mathbf{N}}\right)^{2 n}\right)
$$

exists, where $\left(g_{n}\right)_{n \geq 0}$ is the sequence of (even) moments of a symmetric probability measure $\mu_{d}$ on the real line, depending on $d$. These moments satisfy the following recurrence: $g_{0}=g_{1}=1$ and

$$
g_{n}=\sum_{m=1}^{n} \gamma_{d}(m) g_{m-1} g_{n-m}
$$

for $n \geq 1$, where the coefficients $\gamma_{d}(m)$ are specified for each of the above examples: $\gamma_{d}(m)^{-1}=m^{d}$ in Example 1, $\gamma_{d}(m)^{-1}=\left(\begin{array}{c}m(d+1) \\ d+1\end{array}\right)$ in Example 2, $\gamma_{d}(m)=$ $\left(\frac{d+1}{2} B\left(\frac{d+1}{2} ; \frac{(m-1)(d+1)}{2}\right)\right)^{d}$ in Example 3 (here $B(s+1, t+1):=\int_{0}^{1} x^{s}(1-x)^{t} d x$ is the Euler $\beta$-function), and $\gamma_{d}(1)=1, \gamma_{d}(m)=0$ for $m \geq 2$ in Example 4 .

REMARK 8. The coefficients $\gamma_{d}(m)$ can be computed as the following limit:

$$
\gamma_{d}(m)=\lim _{\mathbf{N} \rightarrow \infty} \sum_{\mathbf{k} \leq \mathbf{N}} \frac{\left|I_{\mathbf{k}}\right|}{\left|J_{\mathbf{N}}\right|}\left(\frac{\left|J_{\mathbf{N}-\mathbf{k}}\right|}{\left|J_{\mathbf{N}}\right|}\right)^{m-1} .
$$

REMARK 9. If $\gamma_{d}(m) \equiv 1$, which is formally a possible case in Example 1 with $d=0$, then the recurrence defines the Catalan numbers, which are moments of the semi-circle law (free CLT). 
REMARK 10. If $\gamma_{d}(m)=\frac{1}{m}$, which is the case in Example 1 for $d=1$, and in Example 2 . for $d=0$, then the recurrence defines the sequence of (even) moments of the arcsine distribution (monotonic CLT).

REMARK 11. In Example 4 we obtain the constant sequence $g_{n} \equiv 1$ of (even) moments of the measure $\mu=\frac{1}{2}\left(\delta_{1}+\delta_{-1}\right)$ (boolean CLT).

\section{References}

[1] M. Bożejko, Positive-definite kernels, length functions on groups and noncommutative von Neumann inequality, Studia Math. 95 (1989), 107-118.

[2] M. Bożejko and J. Wysoczański, Remarks on t-transformations of measures and convolutions, Ann. Inst. H. Poincaré Probab. Statist. 37 (2001), 737-761.

[3] U. Franz, Monotone independence is associative, Inf. Dim. Anal. Quantum Prob. Rel. Top. 4 (2001), 401-407.

[4] R. Lenczewski and R. Sałapata, Discrete interpolation between monotone probability and free probability, Inf. Dim. Anal. Quantum Prob. Rel. Top. 9 (2006), 77-106.

[5] N. Muraki, Monotonic independence, monotonic central limit theorem and monotonic law of small numbers, Inf. Dim. Anal. Quantum Prob. Rel. Top. 4 (2001), 39-58.

[6] J. Wysoczański, Monotonic independence on weakly monotone Fock space, Infin. Dimens. Anal. Quantum Probab. Relat. Top. 8 (2005), 259-275.

[7] J. Wysoczański, Monotonic independence associated with partially ordered sets, Infin. Dimens. Anal. Quantum Probab. Relat. Top. 10 (2007), 17-41.

[8] J. Wysoczański, bm-Central Limit Theorems for positive definite real symmetric matrices, preprint. 